\title{
Wake field flow of a sphere falling in a finite duct
}

\author{
J. Markiewicz, W. R. Matson \\ Department of Physics, DePaul University, Chicago, Illinois, U.S.A.
}

Email address:

jdmarkiewicz@gmail.com (J. Markiewicz),wrmokstate@aim.com (W. R. Matson)

\section{To cite this article:}

J. Markiewicz, W. R. Matson. Wake Field Flow of a Sphere Falling in a Finite Duct. American Journal of Modern Physics. Vol. 2, No. 3, 2013, pp. 98-103. doi: 10.11648/j.ajmp.20130203.11

\begin{abstract}
We report on a simple study involving a single non-Brownian sphere settling under the influence of gravity in a quiescent viscous fluid housed in a finite square duct. Spheres are shown to achieve terminal velocity in a fraction of the time predicted by infinite fluid dynamics. Terminal velocities agree well with right cylinder equations for spheres with diameter-to-width ratios less than 0.45 . The finite chamber length results a two-phase flow and interesting wake field dynamics for spheres with diameter-to-width ratios greater than 0.255 .
\end{abstract}

Keywords: Finite Square Duct; Wake Field Flow; Terminal Velocity

\section{Introduction}

The problem of a single sphere falling under the influence of gravity through an infinite Newtonian fluid is both well known and well understood; however, the influence of impermeable boundaries on the hydrodynamics of single spheres continues to be of interest today[1]. Expressions have been derived for the motion of a single sphere in the presence of a single and parallel plane walls[2, 7]. Vassuer \& Cox, for example, have thoroughly discussed the lateral motion of spheres in the vicinity of plane walls[3]. Other expressions have been developed for a single sphere in a right cylinder[4-6]. The increased resistance experienced by the sphere is a consequence of the no-slip boundary condition at each wall and serves to reduce the terminal velocity of the falling sphere; however, the flow in a right square duct is not directly obtained through superposition of the parallel infinite wall configuration[6]. Furthermore, closing one end of the vertical tube or duct would clearly increase this resistance as a consequence of an upward flow mandated by volume conservation; therefore, the terminal velocity of the falling sphere would be further reduced. The motions of spheres in confined geometries are of significance to many engineering applications, including damped pistons, food \& mineral processing, and oil exploration. Such confined geometries can also be found in microfluidics, as parallel planes and square ducts are easily constructed.

In the present paper, we examine a single acrylic sphere falling along the axis of symmetry of a vertical, square, finite duct under the influence of gravity through a viscous mixture of glycerol and water. The sphere achieves terminal velocity in a fraction of the time predicted by infinite fluid dynamics, and the two-phase flow due to finite chamber length results in unexpected wake field dynamics with large particles. Our results are directly applicable to instances of spherical objects moving in confined geometries and are not readily accessible through numerical simulations due to the asymmetrical boundary conditions.

\section{Experiment}

A sketch of the test chamber is shown in Figure 1. The outer chamber is $18 \mathrm{~cm}$ square and $30 \mathrm{~cm}$ tall with $6 \mathrm{~mm}$ thick acrylic walls. The inner chamber has an interior width of $4.34 \mathrm{~cm}$, is $38 \mathrm{~cm}$ tall, and has $5 \mathrm{~mm}$ thick acrylic walls. Water held at a constant $20.00^{\circ} \mathrm{C}$ flows through the approximately two inch gap between the chambers at approximately 7 liters per minute thereby turning the volume of the chamber over every 40 seconds and maintaining thermal equilibrium. Temperature fluctuations in the regulated water were less than $0.005^{\circ} \mathrm{C}$. This thermal control keeps the thermal convection current to less than 50 microns per second, an order of magnitude smaller than the speed of the slowest sphere used in this experiment, 800 microns per second.

The sample fluid is a homogenous mixture of water, glycerin, and a polymer flake tracer solution. The proportions of glycerin and water are adjusted so that the solvent has density $\rho_{\mathrm{f}}=1.174 \pm .002 \mathrm{~g} \cdot \mathrm{cm}^{-3}$ and viscosity $\eta=$ $19.6 \pm 0.5$ centipoise (cp). The density of the acrylic spheres ranges from $\rho_{\mathrm{s}}=1.18 \pm .01 \mathrm{~g} \cdot \mathrm{cm}^{-3}$ to $\rho_{\mathrm{s}}=1.27 \pm .01 \mathrm{~g} \cdot \mathrm{cm}^{-3}$ 
depending exclusively upon sphere diameter. Density variations within a given size were less than $1 \%$. The diameters of the acrylic spheres ranges from $1 / 16$ inch $(d=0.159 \mathrm{~cm})$ to 1 inch $(\mathrm{d}=2.54 \mathrm{~cm})$ with a deviation in diameter for each sphere of less than $1 \%$. This size range results in $0.0365 \leq$ $\mathrm{d} / \mathrm{W} \leq 0.585$ where $\mathrm{d}$ is the diameter of the spheres and $\mathrm{W}=$ $4.34 \mathrm{~cm}$, the interior width of the square vertical duct. Reynolds numbers ( $\mathrm{Re}$ ) based on diameter and Stokes Velocities ranged from $0.002<\Delta \rho \cdot \mathrm{d} \cdot \mathrm{U}_{0} / \eta<52$; whereas, Reynolds numbers based on diameter and measured velocities range from $0.002<\Delta \rho \cdot d \cdot U / \eta<2$.

Flow visualization is accomplished using flat polymer flakes 300-350 microns in diameter which align their surface area normal vectors orthogonal to the local shear flow. Full height images were illuminated symmetrically by 4 incandescent lamps. Close-up flow visualization was illuminated using co-aligned $1 \mathrm{~mm}$ thick sheets of mono-chromatic laser light, as shown in Figure 2. All image sequences were collected using a 1.2 megapixel single-chip color CCD camera. Frame rate was controlled at the camera by an external digital electronic hardware trigger and varied from 10 frames per second to 2 seconds per frame, depending on sphere velocity, with accuracy $\pm 10^{-6}$ seconds per frame. The fluid is randomized by stirring and subsequently allowed to return to a quiescent state between each successive drop. A vacuum holding and release mechanism was used to release the spheres at 0 velocity without disturbing the quiescent fluid.

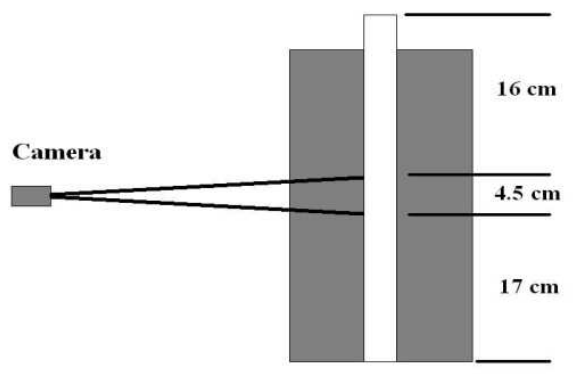

Figure 1. A side view of the apparatus used. The central column is the sample fluid through which the sphere falls under the influence of gravity. The grey fluid surrounding the central column is thermally regulated water set to $20^{\circ} \mathrm{C}$. The camera lens provides a narrow view of a $4.5 \mathrm{~cm} \times 4.5 \mathrm{~cm}$ section of the central column illuminated by a $1 \mathrm{~mm}$ thick laser sheet.

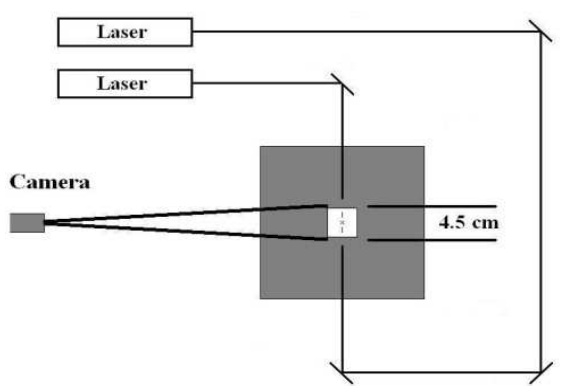

Figure 2. A top view of the apparatus used. The central column is surrounded on all sides with thermally regulated water set to $20^{\circ} \mathrm{C}$. The camera is positioned such that the view width of $4.5 \mathrm{~cm}$ matches the central column width, $4.34 \mathrm{~cm}$. Two co-aligned $1 \mathrm{~mm}$ thick laser sheets, one red \& one green, illuminate the sample from opposing sides.

\section{Observations}

\subsection{Terminal Velocity}

Spheres were dropped from an initial height of $28 \mathrm{~cm}$ and tracked as they traversed the full height of the chamber. Spheres were tracked manually throughout their travel, and velocities were calculated using $\mathrm{U}=\Delta \mathrm{z} / \Delta \mathrm{t}$ where $\Delta \mathrm{z}$ is the displacement in the $\mathrm{x}-\mathrm{z}$ plane and $\Delta \mathrm{t}$ the time between successive frames. Measurements for each size tested were taken for viscosities between $16 \mathrm{cp}<\eta<24 \mathrm{cp}$.

The equations of motion are given by the balance of gravitational and drag forces by Stokes Law:

$$
x^{\prime \prime}(t)+\frac{9 \eta}{2 \rho_{s} a^{2}} x^{\prime}(t)-g\left(\frac{\rho_{f}}{\rho_{s}}-1\right)=0
$$

where $g$ is the acceleration of gravity, $a$ is the radius of the sphere, $\eta$ is the viscosity of the fluid, $\rho_{\mathrm{f}}$ is the density of the fluid, and $\rho_{\mathrm{s}}$ is the density of the sphere. If we define the characteristic time, $\mathrm{t}_{0}$, as

$$
t_{0}=\frac{2 a^{2} \rho_{s}}{9 \eta}
$$

the velocity of a sphere dropped in an infinite fluid as a function of time is found to be

$$
U(t)=\frac{g t_{0}}{\rho_{s}}\left(\rho_{j}-\rho_{s}\right)\left(1-e^{-\frac{t}{t_{j}}}\right)=U_{0}\left(1-e^{-\frac{t}{t_{0}}}\right)
$$

where $\mathrm{U}_{0}$ is the Stokes Velocity.

The 1 inch diameter sphere was tested at viscosities of $16.6 \mathrm{cp}, 19.5 \mathrm{cp}$, and $20.4 \mathrm{cp}$. The measured axial velocities, $\mathrm{U}_{\mathrm{Z}}$, are shown in Figure 3. As expected, wall interactions cause the axial velocities to deviate almost immediately from the infinite fluid prediction, given in Equation 2 and shown by the dotted line in Figure 3. Whereas Equation 1 predicts terminal velocity should be achieved in approximately 12 seconds, Figure 3 shows all trials achieve terminal velocity in 2.5 seconds for each of the viscosities tested. The half-inch sphere was tested at viscosities of 16.6 $\mathrm{cp}, 19.5 \mathrm{cp}$, and $23.4 \mathrm{cp}$. Whereas Equation 2 predicts terminal velocity in approximately 3.5 seconds, Figure 4 shows terminal velocity is achieved between 1.5 and 2.5 seconds. The other diameters each demonstrated similar results, achieving terminal velocity in less than $2.5 \mathrm{~s}$.

In an infinite duct, the time necessary for the flow to become stationary is given by the diffusion of vorticity:

$$
t=\frac{L^{2} \rho_{s}}{\eta}
$$

The length, $\mathrm{L}$, is the distance between the sphere and wall,

$$
L=\frac{4.34 \mathrm{~cm}}{2}-\frac{2.54 \mathrm{~cm}}{2}=0.90 \mathrm{~cm}
$$

so the diffusion time from sphere to wall $\&$ back is 


$$
t=2 \frac{(0.90 \mathrm{~cm})^{2}\left(1.174 \frac{\mathrm{g}}{\mathrm{cm}^{3}}\right)}{0.19 \frac{\mathrm{g}}{\mathrm{cms}}}=10 \mathrm{~s}
$$

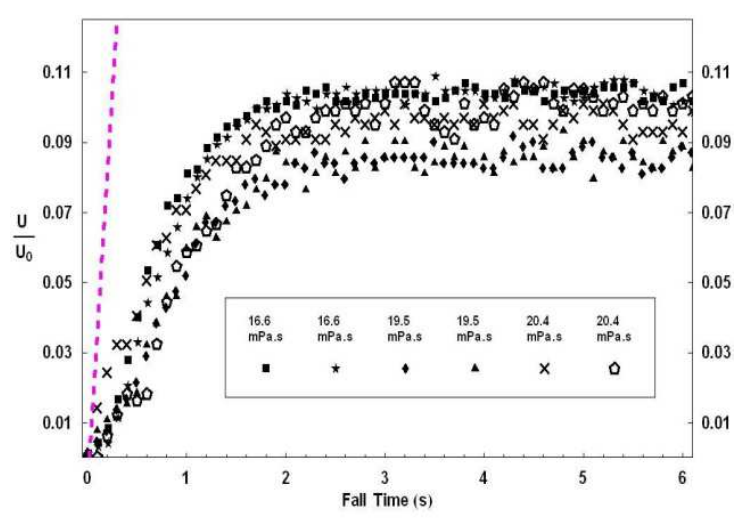

Figure 3. The vertical fall speed, $U$, versus time, $t$, for the 1 inch $(2.54 \mathrm{~cm})$ diameter sphere. The maximum Reynolds number achieved during this trial is $R e=0.17$ based on terminal velocity. The dotted line represents the velocity curve for a similar sphere in an infinite viscous fluid as predicted by Equation 2. Speeds are shown for the first 6 seconds after release for 2 runs each at the following 3 viscosities: $16.6 \mathrm{cp}, 19.5 \mathrm{cp}$, and $20.4 \mathrm{cp}$. In each case, the spheres reach the same terminal velocity within $2.5 \mathrm{~s}$. The terminal velocity is approximately $10 \%$ of the predicted infinite fluid settling velocity, $U_{0}$.

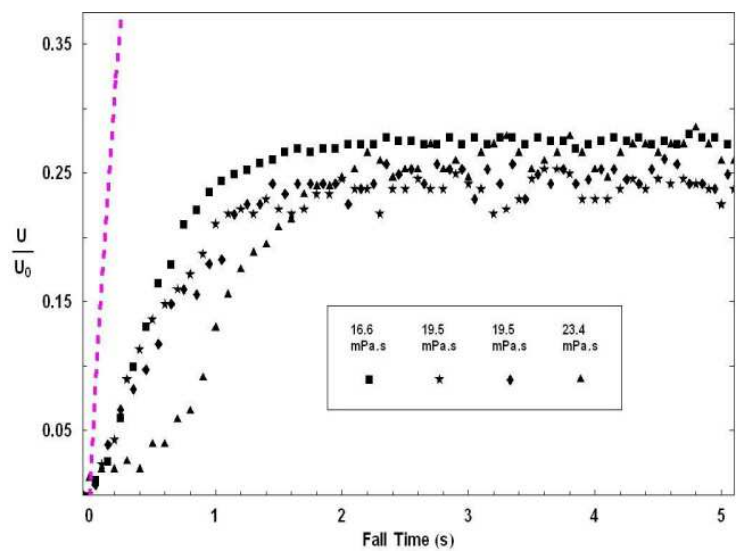

Figure 4. The vertical fall speed, $U$, versus time, $t$, for a half inch $(1.27 \mathrm{~cm})$ diameter sphere. The maximum Reynolds number achieved during this trial is $R e=1.2$ based on terminal velocity. The dotted line represents velocity curve for a similar sphere in an infinite viscous fluid as predicted by Equation 2. Speeds are shown for the first 6 seconds after release for the following 3 viscosities: $16.6 \mathrm{cp}, 19.5 \mathrm{cp}$, and $23.4 \mathrm{cp}$. In each case, the spheres reach the same terminal velocity within $2.5 \mathrm{~s}$. The terminal velocity is approximately $25 \%$ of the predicted infinite fluid settling velocity, $U_{0}$.

However, the duct in this experiment is not infinite. The downward motion of the sphere requires the displacement of fluid past the sphere itself. The fluid cannot move ahead of the sphere, as it would if the duct was infinite. Instead, it must move upward and around the sphere. For smaller spheres, this is not a significant problem; however, the larger spheres occlude a significant percentage of the duct cross section, and the bulk of the flow is compressed into the square corners. This describes a two-phase flow; consequently, the time necessary for the flow to become stationary must be described by the mixing time

$$
t=\frac{L}{U_{\infty}}
$$

where $U_{\infty}$ is the terminal velocity of the system. Therefore, the time for the 1 inch diameter sphere to reach steady state is

$$
t=2 \frac{0.9 \mathrm{~cm}}{1.08 \frac{\mathrm{cm}}{\mathrm{s}}}=1.7 \mathrm{~s}
$$

which is consistent with our laboratory observations and less than the time necessary for the sphere itself to reach terminal velocity.

Axial velocities versus height were also tested to verify terminal velocity was achieved before entering the field of view shown in Figure 1. Axial velocities as a function of height, shown in Figures 5 and 6, illustrate each sphere achieves terminal velocity in less than $20 \%$ of initial height, $5.6 \mathrm{~cm}$, and maintained that speed until less than $10 \%$ of initial height from the chamber floor, or $3 \mathrm{~cm}$. Figure 1 shows the sphere has $16 \mathrm{~cm}$ of travel before entering the front camera view; furthermore, the sphere is still $17 \mathrm{~cm}$ from the chamber floor when it leaves the camera view. These distances are sufficient to ensure the sphere achieves terminal velocity well before entering throughout the field of view, and maintains terminal velocity well after leaving the field of view. This independently verifies measurements on the field of view itself, where the sphere's initial velocity upon entering the field of view and final velocity as it leaves the field of view differed by less than a pixel and was, therefore, immeasurable. Even if we add an additional $1.7 \mathrm{~s}$ for the mixing to occur at terminal velocity, the sphere has only traveled $5.6 \mathrm{~cm}+1.8 \mathrm{~cm}=7.4 \mathrm{~cm}$, which is less than half the distance from the chamber top to the top of the viewing window. Therefore, even accounting for mixing, the flow is clearly in steady state well before entering the viewing area.

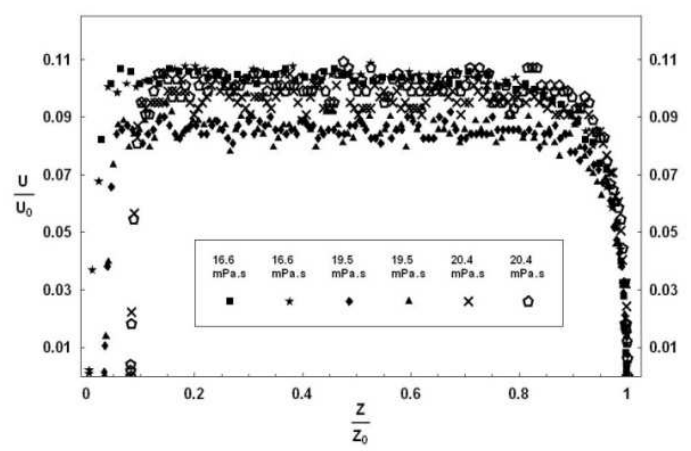

Figure 5. The vertical fall speed versus Z-Height for the 1 inch $(2.54 \mathrm{~cm})$ diameter sphere. The maximum Reynolds number achieved during this trial is $R e=0.17$ based on terminal velocity. The bottom of the chamber is taken as $z=0$, so spheres are falling Right-to-Left. The initial height is set to $z_{0}=$ $28 \mathrm{~cm}$ in each case. Speeds are shown for $z=z_{0}$ to $z=0$ for 2 runs each at the following viscosities: $16.6 \mathrm{cp}, 19.5 \mathrm{cp}$, and $20.4 \mathrm{cp}$. In each case, the spheres reach the same terminal velocity within $20 \%$ of $z_{0}$, or approximately $6 \mathrm{~cm}$, and maintain terminal velocity until they are within approximately $10 \%$ of $z_{0}$, or $3 \mathrm{~cm}$, of the bottom. The terminal velocity of each sphere is approximately $10 \%$ of the infinite fluid settling velocity, $U_{0}$. 


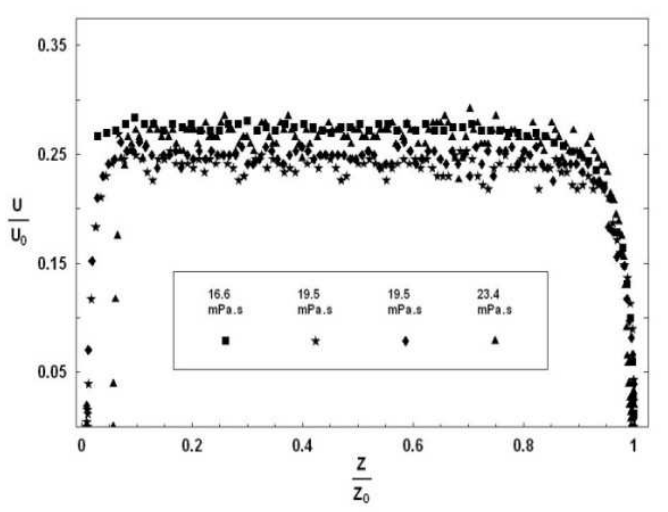

Figure 6. The vertical fall speed versus Z-Height for the $1 / 2$ inch $(1.27 \mathrm{~cm})$ diameter sphere. The maximum Reynolds number achieved during this trial is $R e=1.2$ based on terminal velocity. The bottom of the chamber is taken as $z=0$, so spheres are falling Right-to-Left. The initial height is set to $z_{0}=28 \mathrm{~cm}$ in each case. Speeds are shown for $z=z_{0}$ to $z=0$ for 2 runs each at the following viscosities: $16.6 \mathrm{cp}, 19.5 \mathrm{cp}$, and $20.4 \mathrm{cp}$. In each case, the spheres reach the same terminal velocity within $20 \%$ of $z_{0}$, or approximately $6 \mathrm{~cm}$, and maintain terminal velocity until they are within approximately $10 \%$ of $z_{0}$, or $3 \mathrm{~cm}$, of the bottom. The terminal velocity of each sphere is approximately $25 \%$ of the infinite fluid settling velocity, $U_{0}$.

\subsection{Reverse Wake Flow}

Volume conservation in this closed container requires the sphere to displace the fluid beneath it as it falls, thereby generating an upward flow in the chamber. Smaller spheres have both less volume and less velocity so the unoccupied region between sphere and wall is more than sufficient to handle the upward flow; however, spheres with diameters $\mathrm{d} / \mathrm{W}>0.255$ displace fluid faster due to increased sphere volume and mass. The region between sphere and wall is also smaller, and the upward flow is compressed into the corners to minimize viscous losses. We believe the corner flows converge behind the sphere due to decreased local hydrodynamic pressure and form the upward flowing region in the wake of the sphere shown in Figure 7. The upward flow in the wake is approximately 1 sphere radius in diameter.

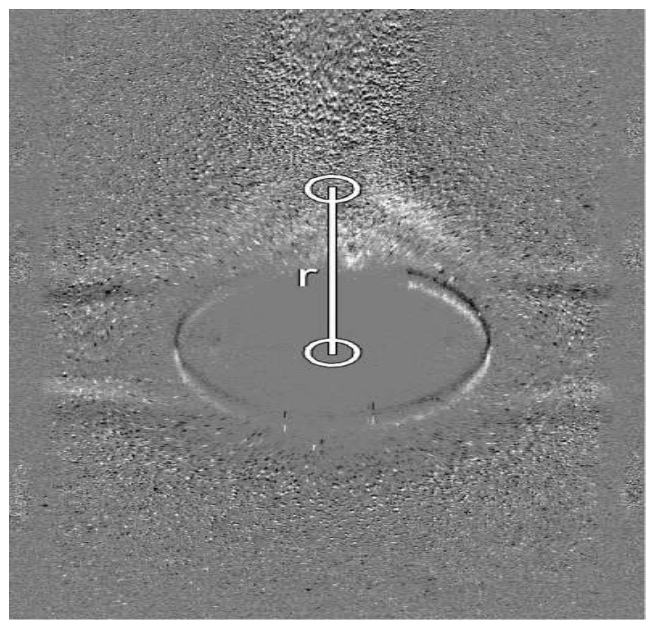

(a)

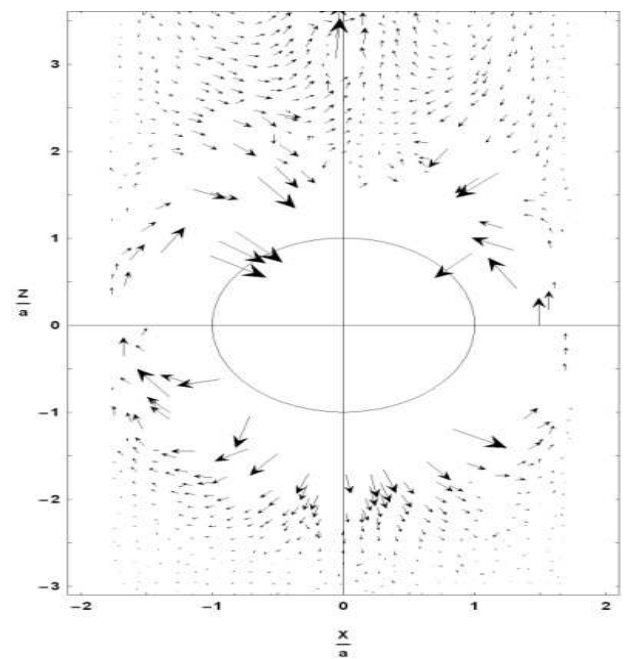

(b)

Figure 7. The top is picture is a differential image created by comparing adjacent frames in a movie sequence indicating the flow field around a $2.54 \mathrm{~cm}$ sphere, $d / W=0.585$. Motion indicated in this image is directed from Black toward White. The bottom picture is a vector field plot created using particle image velocimetry on the same movie sequence. The circle has been added to represent the location of the sphere and provide a point of reference. The longest vectors are equivalent to the terminal velocity of the sphere, $1.08 \mathrm{~cm} / \mathrm{s}$. The Reynolds number based on diameter and terminal velocity is $R e=0.17$. The distance $r$ is measured from the sphere center to a stagnation point indicated behind the sphere where magnitude velocity drops near 0 . In this case, the distance $r / a \approx 2.1$, where $a$ is the sphere radius. $A$ " $V$ " formation shows the upward moving fluid. The width of this column is approximately 1 sphere radius. The magnitude velocity is approximately $10 \%$ of the terminal velocity of the sphere.

Particle image velocimetry measurements were taken for each sphere. The velocity vectors for a 1 inch diameter sphere are shown in Figure 7 adjacent to a differential image from the same sequence for comparison. Using these velocimetry measurements, central velocities were determined by considering all velocity vectors within $\sqrt{x^{2}+y^{2}} \leq \mathrm{a} / 2$ at intervals of $\Delta \mathrm{z}=0.50 \mathrm{~mm}$. For each interval an average axial velocity is determined. The average central velocities for the three largest spheres are shown in Figure 8. In each case, the central velocity approaches the sphere velocity as $\mathrm{r} / \mathrm{a} \rightarrow 1$, as expected. The central velocity decreases exponentially until it crosses the horizontal axis. The velocity ratio becomes negative beyond this point indicating the flow is traveling opposite to that of the sphere. The location of these stagnation points are given in Figure 9 as a function of size. The distance from the sphere center to the convergence point, r/a, depends non-linearly upon the size of the sphere. This may be a consequence of increased mass, speed, and volume displacement of the sphere correlated with the cross-sectional area of the duct. The dashed vertical line approximates the critical diameter taken to be halfway between 0.219 and 0.292 , or $\mathrm{d} / \mathrm{W} \approx 0.255$. 


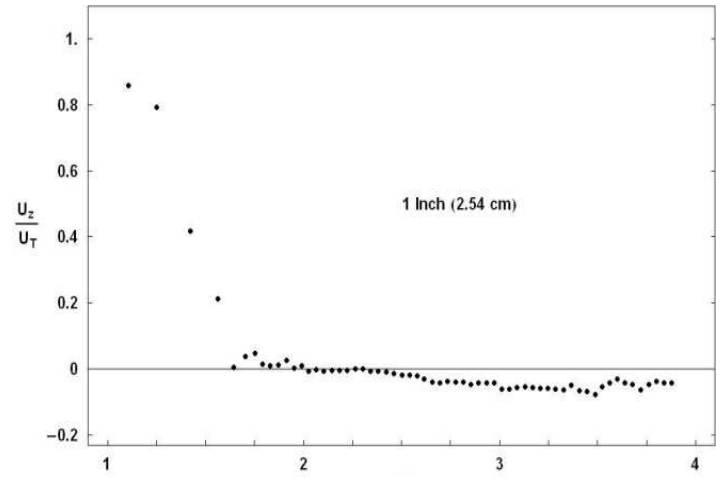

(a)

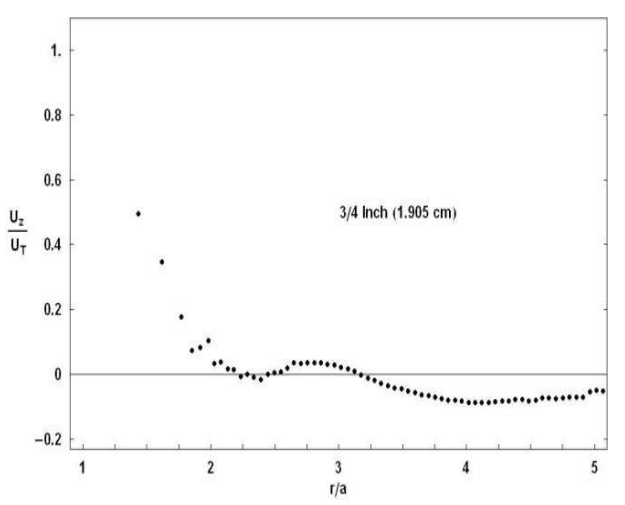

(b)

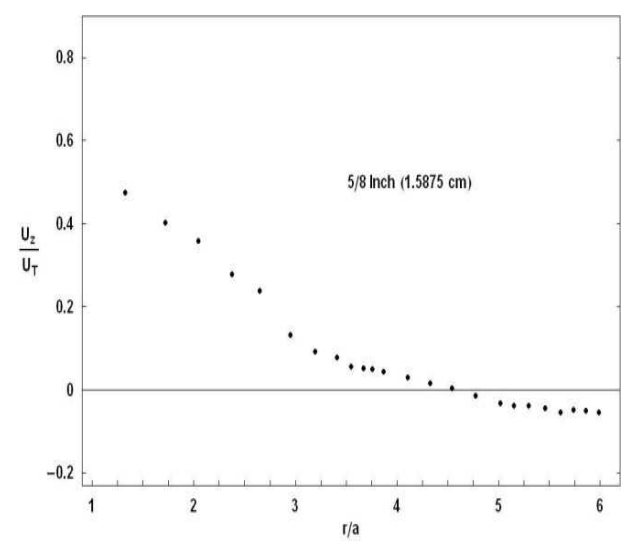

(c)

Figure 8. The average dimensionless central axial velocity, $U_{Z} / U_{T}$, as a function of distance from sphere center. Averages are taken over a region of $-a / 2<x<a / 2$ and heights $\Delta z=0.50 \mathrm{~mm}$ based on velocimetry measurements in that area where $a$ is the radius of the sphere. The value $r / a==1$ equates to the top edge of the sphere, and $U_{Z} / U_{T}==1$ equates to terminal velocity, $U_{T}$. The Reynolds Numbers based on diameter and terminal velocity are: $(A) 0.17,(B) 0.18$, and (C) 1.8. The stagnation point in the wake field is indicated where the velocities cross the horizontal axis, hence changing vertical direction.

While the Reynolds number based on measured velocity for the flow shown in Figure 7 corresponds to $\operatorname{Re}=0.17$, no clear correlation between r/a and the Reynolds number is noted. The largest measurement, $\mathrm{r} / \mathrm{a}=12$, occurs at the value $\operatorname{Re}=1.2$; whereas, the largest Reynolds number of $\operatorname{Re}=1.8$ yields $\mathrm{r} / \mathrm{a}=4.5$ and corresponds to the sphere shown in Figure 8-C.

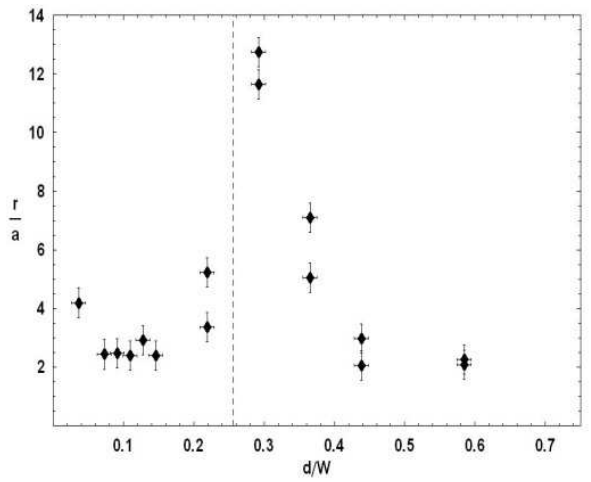

Figure 9. Dimensionless distance $r / a$ versus the diameter ratio $d / W$, where $r$ is the measured distance from sphere center to the stagnation point preceding the start of upward fluid velocity (see Figure 7), $a$ is the radius of the sphere, $d$ is the diameter of the sphere, and $W$ is the inner width of the square duct. The dashed vertical line indicates the estimated critical ratio, $d / W \approx 0.255$. Horizontal error bars are based on the error in size, $0.01 \mathrm{~cm}$, and vertical error bars are based on the accuracy in $r$ shown in Figure 7.

\subsection{Finite Terminal Velocity}

The terminal velocities measured agree well with values predicted by equations for right circular cylinders up to $\mathrm{d} / \mathrm{W}$ values of 0.45 , even though the geometry is square, as shown in Figure 10. A round cylindrical chamber requires $\mathrm{U} / \mathrm{U} 0 \rightarrow 0$ as $\mathrm{d} / \mathrm{W} \rightarrow 1$; however, in a non-circular chamber, $\mathrm{U} / \mathrm{U} 0$ approaches some non-zero limit as $\mathrm{d} / \mathrm{W} \rightarrow 1$, as noted by Pitois, Pasol, \& Vignes-Adler[8]. Our results in Figure 10 suggests a limit $\mathrm{U} / \mathrm{U} 0 \approx 0.2$ for a right square duct which is in good agreement with the value 0.25 reported by Pitois.

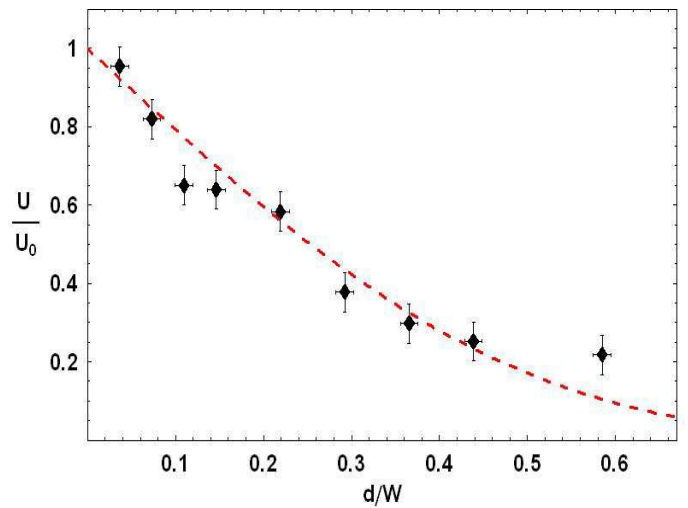

Figure 10. Dimensionless settling Velocity U/UO versus the diameter ratio $d / W$. U is the measured settling velocity, $U 0$ is the Stokes Velocity, $d$ is the diameter of the sphere, and $W$ is the inner width of the right cylindrical chamber. The dashed line represents the theoretical velocity of a sphere falling along the axis of symmetry in a right circular cylinder. Horizontal error bars are based on the error in size, $0.01 \mathrm{~cm}$, and vertical error bars are based on the variance in terminal velocity as in Figures 5 \& 6 .

\section{Conclusion}

We have examined spheres falling in a vertical square duct. In each case, spheres achieve terminal velocity in less than $2.5 \mathrm{~s}$ from release during which time they travel less than $6 \mathrm{~cm}$. Terminal velocity is found to agree well with the 
right cylinder velocity equations for diameter-to-width ratios $\mathrm{d} / \mathrm{W}<0.45$. Our results suggest a finite terminal velocity of $\mathrm{U} / \mathrm{U} 0 \approx 0.2$ as $\mathrm{d} / \mathrm{W} \rightarrow 1$, which agrees well with the value 0.25 reported by Pitois. Spheres with $\mathrm{d} / \mathrm{W}>0.255$ demonstrate a wake flow traveling in the opposite direction of the sphere about the axis of symmetry of the duct, one sphere radius in diameter. The point where the flow reverses direction varies non-linearly with sphere diameter.

\section{References}

[1] J. W. Swan \& J. F. Brady, "Particle motion between parallel walls: Hydrodynamics and simulation" Phys. Fluids. 22, 103301 (2010)

[2] L. Zovatto \& G. Pedrizzetti, "Flow about a circular cylinder between parallel walls" J. Fluid Mech. 440 (2001)

[3] P. Vasseur \& R. G. Cox, "The lateral migration of spherical particles sedimenting in a stagnant bounded fluid" J. Fluid
Mech. 80 (1977)

[4] T. Bohlin, "On the drag on a rigid sphere moving in a viscous liquid inside a cylindrical tube" Transactions of the Royal Institute of Technology 155 (1960)

[5] W. L. Haberman \& R. M. Sayre, "Motion of rigid and fluid spheres in stationary and moving liquids inside cylindrical tubes" David Taylor Model Basin Report \#1143, U. S. Department of the Navy (1958)

[6] J. Happel \& E. Bart, "The settling of a sphere along the axis of a long square duct at low Reynolds' number" Applied Scientific Research 29, 1 (1974)

[7] A. Miyamura, S. Iwasaki, \& T. Ishii, "Experimental wall correction factors of single solid spheres in triangular and square cylinders, and parallel plates" International Journal of Multiphase Flow 7, 1 (1981)

[8] O. Pitois, C. Fritz, L. Pasol, \& M. Vignes-Adler, "Sedimentation of a sphere in a fluid channel" Phys. Fluids 21103304 (2009) 